\title{
Intravenous Paracetamol Versus Patient-Controlled Analgesia With Morphine for the Pain Management Following Diagnostic Knee Arthroscopy in Trauma Patients: A Randomized Clinical Trial
}

\author{
Seyed Masoud Hashemi, ${ }^{1}$ Aliakbar Esmaeelijah, ${ }^{2}$ Samad Golzari, ${ }^{3}$ Sohrab Keyhani, ${ }^{2}$ Azita \\ Maserrat, ${ }^{4}$ Gholamreza Mohseni, ${ }^{4, *}$ and Seyed Hosein Ardehali ${ }^{4}$ \\ ${ }^{1}$ Department of Pain Management, Shahid Beheshti University of Medical Sciences, Tehran, IR Iran \\ ${ }_{3}^{2}$ Department of Orthopedics, Shahid Beheshti University of Medical Sciences, Tehran, IR Iran \\ ${ }^{3}$ Department of Anesthesiology, Tabriz University of Medical Sciences, Tabriz, IR Iran \\ ${ }^{4}$ Department of Anesthesiology, Shahid Beheshti University of Medical Sciences, Tehran, IR Iran \\ ${ }^{*}$ Corresponding author: Gholamreza Mohseni, Department of Anesthesiology, Shahid Beheshti University of Medical Sciences, Tehran, IR Iran. Tel: +98-9375347941, \\ E-mail:aramgol@gmail.com
}

Received 2015 June 15; Revised 2015 August 19; Accepted 2015 September 2.

\begin{abstract}
Background: Most patients undergoing outpatient surgeries have the unpleasant experience of high level pain after surgery. Compared with open surgeries, arthroscopic procedures are less painful; however, inadequate pain management could be associated with significant concerns. Opioids alone or in combination with local anesthetics are frequently used for diminishing postoperative pain using intravenous or epidural infusion pumps. Despite morphine various disadvantages, it is commonly used for controlling pain after surgery. Objectives: The aim of this study was to compare intravenous paracetamol and patient-controlled analgesia (PCA) with morphine for the pain management following diagnostic knee arthroscopy in trauma patients.

Patients and Methods: Sixty trauma patients who were scheduled to undergo knee arthroscopy were randomly divided into two groups. Patients immediately received intravenous infusion of $1 \mathrm{~g}$ paracetamol within 15 minutes after surgery and every 6 hours to 24 hours in the paracetamol group. The patient-controlled analgesia group received morphine through PCA infusion pump at $2 \mathrm{~mL} / \mathrm{h}$ base rate and $1 \mathrm{~mL}$ bolus every 15 minutes. Pain level, nausea and vomiting, and sedation were measured and recorded during entering the recovery, 15 and 30 minutes after entering the recovery, 2, 6, and 24 hours after starting morphine pump infusion in the morphine and paracetamol in the paracetamol groups.

Results: There was no significant difference regarding the pain level at different times after entering the recovery between the two groups. No one from the paracetamol group developed drug complications. However, $22.3 \%$ in the PCA morphine suffered from postoperative nausea; there was a statistically significant difference regarding the sedation level, nausea, and vomiting at various times between the two groups.

Conclusions: Intravenous administration of paracetamol immediately after knee arthroscopy improved postoperative pain, decreased analgesic administration, maintained stable hemodynamic parameters, had no complications related to opiates, no nausea and vomiting, and increased patient satisfaction and comfort in comparison to PCA with morphine.
\end{abstract}

Keywords: Analgesia, Patient-Controlled, Patient Safety, Pain Management, Arthroscopy, Acetaminophen, Morphine

\section{Background}

Patient safety has always been a major concern for the physician in all eras (1) and even ancient physicians used their own methods to reduce pain of the patients (2). Recent advances in surgical techniques and anesthesia have increased the number of surgeries in outpatient setting worldwide. Consequently, more than $60 \%$ of patients in the West are outpatient surgery cases (3). Diagnostic and therapeutic arthroscopies are common procedures for outpatient surgeries under general anesthesia or regional anesthesia. Compared with open surgeries, arthroscopic procedures are less painful; however, inadequate pain management could be associated with signifi- cant concerns. Cost-effectiveness of outpatient surgeries is well-known.

Effective postoperative pain controlling is necessary and important for optimizing patients comfort and satisfaction. Furthermore, providing a rapid recovery and reducing pain and discomfort for controlling pain after outpatient surgery is necessary to improve the patient outcome. Different analgesic techniques from multimodal approaches $(4,5)$ to blocks $(6,7)$, corticosteroids $(7,8)$, and local anesthetics (9) have been introduced. Analgesics administration is growing up in outpatient surgeries in order to prevent postoperative pain. Inad-

Copyright (C) 2015, Kashan University of Medical Sciences. This is an open-access article distributed under the terms of the Creative Commons Attribution-NonCommercial 4.0 International License (http://creativecommons.org/licenses/by-nc/4.0/) which permits copy and redistribute the material just in noncommercial usages, provided the original work is properly cited. 
equate control of pain after surgery is one of the key factors for failing to discharge patients $(3,10,11)$. Postoperative pain control improves the patient's ability and increases their performance in their daily life activities (12).

Most patients undergoing outpatient surgeries have the unpleasant experience of high level pain after surgery $(3,10,11)$. About $30 \%-40 \%$ of the outpatient surgery candidates experience moderate to severe pain during first 24 - 48 hours after surgery (13-15). In some cases, the pain may become more severe over time and affect sleep and daily activities $(16,17)$. Moreover, the duration and type of surgery have a significant effect on pain severity which may ultimately lead to further analgesic requirements $(13,15,18)$. Different techniques of anesthesia and analgesia have their own advantages and disadvantages. Patient-controlled analgesia (PCA) pump is one of the pain management methods in which the patient manages the pain using programmed infusion and further analgesic boluses. Drug infusion pump is set according to patient's needs to maintain adequate analgesia. Different groups of analgesics exert their effects through different mechanisms. Opioids are gold-standard analgesics for postoperative analgesia; however, due to their undesirable side effects, such as apnea, urinary retention, nausea, and vomiting can lead to patient discomfort. Opioids alone or in combination with local anesthetics are frequently used for diminishing postoperative pain using Intravenous or epidural infusion pumps. Despite morphine various disadvantages, it is commonly used for controlling pain after surgery. Onset time for intravenous morphine is $1-2$ minutes after injection; its peak effect is 3 - 5 minutes after injection and the duration of its analgesic effect is 1-2 hours (18). Opioids and none-steroids anti-inflammatory drugs (NSAIDs) are the main analgesic drugs that are commonly used to control pain after surgery.

Other strategies are recently implemented to improve postoperative pain control and decline the side effects associated with opioids. Intravenous paracetamol (acetaminophen) is one of the new strategies used for improving mild to moderate pain intensity (19). With an onset of action of 5-10 minutes after the injection, acetaminophen displays a peak effect one hour after injection and its analgesic effects endure for 4 - 6 hours (18-20). Paracetamol is used for managing mild to mediocre pains (20).

\section{Objectives}

The present study was conducted to compare Intravenous paracetamol and patient controlled analgesia with morphine for the pain management following diagnostic knee arthroscopy in Trauma patients.

\section{Patients and Methods}

This randomized clinical trial was conducted on 60 eligible trauma patients who were scheduled for under- going diagnostic knee arthroscopy at Akhtar hospital in Tehran city, Iran, during October 2013 to October 2014.

Sample size was acquired using the two means comparison formula. The power value was determined to be $80 \%$, with an assumed dropout rate of $20 \%$. Considering $d=4$, sample size of 30 for each group was obtained.

Before recruitment of first subject, study protocol was approved by local ethics committee of Shahid Beheshti University of medical sciences. The study has been performed in accordance with the ethical standards of the 1964 Declaration of Helsinki. All patients signed the informed consent forms prior to recruitment in the study.

The sample consisted of sequential selection of $60 \mathrm{pa}-$ tients who were divided consecutively and randomly into two groups (intravenous paracetamol or morphine PCA pumps).

Inclusion criteria were: trauma patients undergoing diagnostic knee arthroscopy under spinal anesthesia, patients between 15 - 60 years of age, American society of anesthesiology classification (ASA) I-II, lack of other coexisting diseases and lack of history of neurologic drugs use.

Exclusion criteria were: contraindications of spinal anesthesia, history of analgesic medication 24 hours before surgery, inability to use the PCA, history of bleeding or coagulopathies within the previous month, liver or kidney failure, severe cardiopulmonary, obesity (body mass index $>30 \mathrm{~kg} / \mathrm{m}^{2}$ ), history of postoperative nausea and vomiting, history of migraine, history of complications during or after surgery, postoperative complications during 24 hours after surgery requiring intervention, known allergy, allergy or contraindications to opioid analgesics or nonopioid drugs, pregnancy or lactation, and history of alcohol or drug abuse.

All the patients were operated with the simultaneous presence of the same surgeon and anesthetist and the same surgical routine. The duration of the procedure was considered to be 30 to 40 minutes and in case of any complication which would make it longer, the patients were excluded from the study.

All the patients first received preanesthesia consisting of $50 \mathrm{mg}$ of fentanyl and $1 \mathrm{mg}$ of midazolam.

Blood pressure and heart rate were measured and recorded by Non invasion Blood Pressure (NIBP) monitoring and a pulse oximetry.

Spinal anesthesia was made by a puncture in the L4-L5 intervertebral space and infusion of $10 \mathrm{mg}$ of $0.5 \%$ isobaric Bupivacaine $®$.

Systolic pressure decline of more than $30 \%$ of base level or less than $100 \mathrm{mmHg}$ was considered as hypotension which was treated by infusion of intravenous fluids and $6 \mathrm{mg}$ of ephedrine every 2 minutes until reaching normal blood pressure levels. Decrease of heart rate to less than 60 beats per minute was considered a major change, which was treated by the administration of $0.5 \mathrm{mg}$ of intravenous atropine.

After the procedure, patients of the first group received 
intravenous infusion of $1 \mathrm{~g}$ paracetamol (Apotel; UniPharma. SA, India) within 15 minutes after surgery and every 6 hours to 24 hours in the paracetamol group.

At the same time, The PCA group received morphine through PCA infusion pump at $2 \mathrm{~mL} / \mathrm{h}$ base rate and $1 \mathrm{~mL}$ bolus every 15 minutes.

Pain level, nausea and vomiting and sedation were measured and recorded during entering the recovery, 15 and 30 minutes after entering the recovery, 2, 6, and 24 hours after starting morphine pump infusion in the morphine and paracetamol in the paracetamol groups.

The pain score was measured based on visual analogue scale (VAS) scale ( $0=$ no pain, $1-3=$ mild pain, $4-7=$ moderate pain, and $8-10=$ severe pain). If VAS was more than 3, $2 \mathrm{mg}$ bolus morphine was injected.

Side effects of morphine were assessed every 4 hours in the first 24 hours after surgery. The drugs used in the first 24 hours after surgery, possible side effects of paracetamol (rash, thrombocytopenia, leukopenia, hypotension, and renal or liver failure), and patient analgesia satisfaction quality after 24 hours were evaluated based on VAS (zero = dissatisfaction, 1 - 3 = medium satisfaction, 4 - 6 = good satisfaction, and $7-10=$ high satisfaction).

Further morphine requirement based on $\mathrm{mg}$, sedation score based on the Ramsay scale (21) (1-anxious, agitated, 2-quiet, alert, 3-sleepy, 4-dizzy with response to verbal commands, 5-no response to verbal commands, 6-no response to painful stimuli), and nausea and vomiting (22) ( 1 = no nausea, vomiting, 2 = nausea, $3=$ once or twice nausea and vomiting, 4 = nausea and vomiting more than twice) were studied at different times.

The assessment was performed in recovery and in the ward by trained nursing staff who were not part of the team for the present study.

Data were analyzed using SPSS 19 (SPSS Inc, Chicago, IL, USA). Comparisons between the groups were made using Chi-square or Fisher' s exact test when appropriate and $P$ value less than 0.05 was considered statistically significant.

\section{Results}

In the present study, 30 patients (50\%) were in the paracetamol and 30 patients (50\%) in the PCA morphine groups. Comparison of demographic data between the groups showed no significant statistical difference (Table 1).

Changes in pain score between the two groups at different times are demonstrated in Figure 1. There was no significant difference regarding the pain level at different times after entering recovery between two groups ( $\mathrm{P}=$ 0.076).

Recovery time in the paracetamol and PCA morphine, administered further analgesic (morphine), the time for receiving first dose of opioids were compared and analyzed. None of the mentioned outcome measures showed a statistically significant difference between the two groups $(\mathrm{P}>0.05)$ (Table 2$)$.
No one from the paracetamol group developed drug complication. Nevertheless, 7 patients $(22.3 \%)$ in the PCA morphine suffered from postoperative nausea; there was a statistically significant difference between the two groups in this regard $(\mathrm{P}=0.005)$.

There was a statistically significant difference regarding the sedation level at various times between the two groups. Furthermore, there was a statistically significant difference regarding nausea and vomiting at the various times between the two groups. Moreover, there was a statistically significant difference regarding patient satisfaction between the two groups $(\mathrm{P}=0.0001)$.

Table 1. Demographic Variables Compared Between the Two Groups $^{\mathrm{a}}$

\begin{tabular}{lccc}
\hline & Paracetamol & PCA Morphine & P Value \\
\hline Age, $\mathbf{y}$ & $38.5 \pm 1.6$ & $40.3 \pm 2.4$ & 0.647 \\
Weight, kg & $68.7 \pm 13.2$ & $68.1 \pm 4.1$ & 0.803 \\
\hline Gender & & & 0.543 \\
\hline Male & $20(66.7 \%)$ & $22(73.3 \%)$ & \\
\hline Female & $10(33.3 \%)$ & $8(26.7 \%)$ & \\
ASA class & & & 0.216 \\
\hline I & $22(33.3 \%)$ & $19(28.8 \%)$ & \\
\hline II & $8(33.3 \%)$ & $11(45.8 \%)$ & \\
\hline
\end{tabular}

a Abbreviations: ASA, American society of anesthesiology; PCA, patient controlled analgesia.

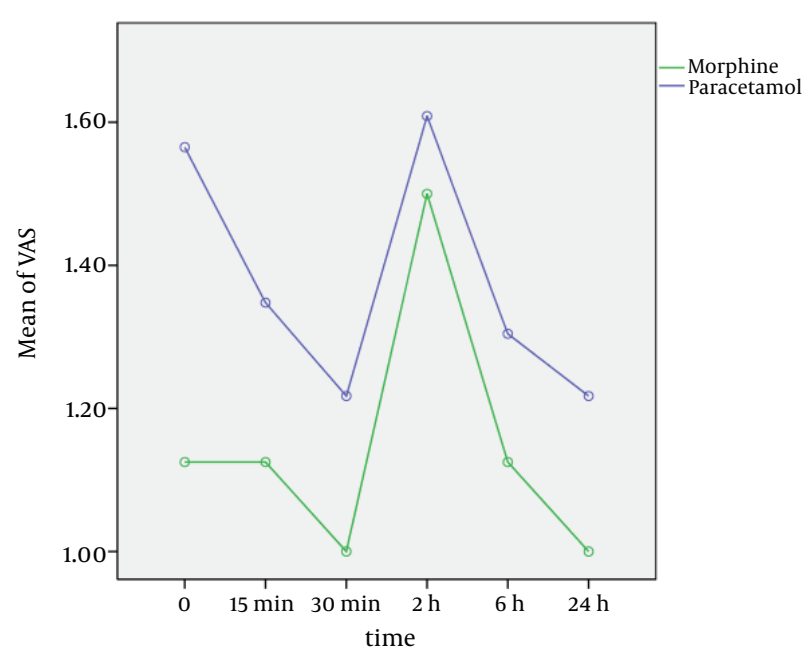

Figure 1. Pain Score in Two Groups at Different Times 
Hashemi SM et al.

\begin{tabular}{lccc}
\hline \multicolumn{1}{l}{ Table 2. Outcome Measures Compared Between the Two Groups ${ }^{\mathrm{a}, \mathrm{b}}$} & & \\
\hline & Paracetamol & PCA & P Value \\
\hline Recovery time, min & $34.0 \pm 5.1$ & $33.1 \pm 0.4$ & $>0.05$ \\
Extra analgesic, mg & $0.5 \pm 0.0$ & $1.0 \pm 0.5$ & $>0.05$ \\
$\begin{array}{l}\text { Time of first request for } \\
\text { opioids, min }\end{array}$ & $15.0 \pm 0.9$ & $16.0 \pm 9.7$ & $>0.05$ \\
drug complications & 0 & $7(23 \%)$ & $<0.05$ \\
\hline a & & &
\end{tabular}

abbreviation: PCA, patient controlled analgesia.

$\mathrm{b}_{\mathrm{N}}=30$.

\section{Discussion}

Effective control of postoperative pain is a major concern for anesthesiologists. Despite the development of improved methods of pain control, patients still suffer from inadequate postoperative pain management. Effective control of postoperative pain by opioids has several side-effects such as nausea, vomiting, pruritus, urinary retention and respiratory depression (23). Therefore, the use of non-opioids has become more popular to avoid opioid-related side-effects (24).

In this study, changes in pain scores between the two groups depicted no significant difference; during the 24 hours after surgery, paracetamol and morphine were similarly reduced the severity of postoperative pain. The pain severity 24 hours after operation was mild and none of the patients had pain in both paracetamol and morphine groups. Intravenous paracetamol has been reported to provide effective analgesia and pain control and consequently reduced opioid consumption after breast surgery outpatients. Reducing opiates consumption is very important because it leads to a decrease in side-effects and life-threatening complications of opioids. Admittedly, any therapy that reduces total opioid administration dose is considered an advantage. The results of this study are consistent with previous studies in which paracetamol declines the administration of opiates (25-27).

In the clinical studies, one gram of intravenous paracetamol has been reported to be as effective as Ketorolac $30 \mathrm{mg}$, $75 \mathrm{mg}$ diclofenac, and morphine $10 \mathrm{mg}$. It is beneficial for the treatment of moderate to severe pain after surgery $(28,29)$. Sinatra et al. study showed that one gram of intravenous paracetamol in patients with moderate to severe pain resulted in rapid and effective analgesia in the first 24 hours after orthopedic surgeries (30). Another study showed that nonopioid analgesics are beneficial as well as opioids in controlling pain after endonasal surgery (31).

The exact mechanism of action of paracetamol has not been determined. Paracetamol may lead to the activation of serotonin receptor II and inhibition of cyclooxygenase pathway in the synthesis of prostaglandins with doublebarreled effect (32-34). Paracetamol may be associated with hemorrhagic side-effects by inhibiting the synthe- sis of prostaglandins and related compounds which has only been reported for NSAIDs (32). There was no bleeding case in the present study.

All of the paracetamol patients were calm and alert at discharge while 7 patients of the PCA morphine were anxious, agitated, and restless 15 and 30 minutes after entering the recovery. This may be due to insufficient control of pain in the paracetamol group. In the present study, no significant changes in systolic blood pressure, diastolic blood pressure, means arterial blood pressure, and heart rate were observed in the paracetamol group. Paracetamol may decrease cardiac output and peripheral vascular resistance (35). In our study, systolic and diastolic blood pressure, and mean arterial blood pressure decline was $5 \mathrm{mmHg}$ and blood pressure was maintained within the normal range; none of patients developed hypotension.

Also, in the morphine pump group, imbalance in hemodynamic was observed frequently; while, the paracetamol group patients experienced a further stable hemodynamics.

The results obtained from our study presented that intravenous administration of one gram of paracetamol in diagnostic arthroscopic knee surgery immediately after the surgery has clinical benefits such as improved postoperative pain, decreasing of analgesics administration, stable hemodynamic parameters, reducing length of hospitalization in the recovery room, lack of complications related to opiates, no nausea and vomiting, and increased patient satisfaction and comfort. Conducting studies with different doses of paracetamol, different surgical processes and larger sample sizes are recommended for the future studies.

\section{Footnote}

Authors' Contribution:Seyed Masoud Hashemi had primary responsiblity for protocol development, patient screening, enrollment, outcome assessment, preliminary data analysis and writing the manuscript. Aliakbar Esmaeelijah and Samad Golzari participated in the development of the protocol and analytical framework for the study and contributed to the writing of the manu- 
script. Azita Maserrat contributed in the development of the protocol and analytical framework for the study and contributed to the writing of the manuscript and was responsible for patient screening. Gholamreza Mohseni supervised the design and execution of the study, performed the final data analyses and contributed to the writing of the manuscript.

\section{References}

1. Golzari SE, Khan ZH, Ghabili K, Hosseinzadeh H, Soleimanpour $\mathrm{H}$ Azarfarin R, et al. Contributions of Medieval Islamic physicians to the history of tracheostomy. Anesth Analg. 2013;116(5):1123-32. doi:10.1213/ANE.ob013e3182884313. [PubMed: 23492962]

2. Dabbagh A, Rajaei S, Golzari SE. History of anesthesia and pain in old Iranian texts. Anesth Pain Med. 2014;4(3):e15363. doi: 10.5812| aapm.15363. [PubMed: 25237631]

3. Chauvin M. State of the art of pain treatment following ambulatory surgery. Eur J Anaesthesiol Suppl. 2003;28:3-6. [PubMed: 12785455]

4. Aghamohammadi D, Hosseinzadeh $\mathrm{H}$, Eidy M, Mohammadzadeh Vizhe Z, Abolghasemi Fakhri MB, Movassaghi R, et al. Multimodal preincisional premedication to prevent acute pain after cholecystectomy. J Cardiovasc Thorac Res. 2012;4(3):65-8. doi: 10.5681/ jcvtr.2012.016. [PubMed: 24250987]

5. Soleimanpour H, Gahramani K, Taheri R, Golzari SE, Safari S, Esfanjani RM, et al. The effect of low-level laser therapy on knee osteoarthritis: prospective, descriptive study. Lasers Med Sci. 2014;29(5):1695-700. doi: 10.1007/s10103-014-1576-6. [PubMed: 24733283]

6. Aghamohamadi D, Hosseinzadeh H, Golzari SE, Alizadeh A, Peirovifar A, Movassagi R, et al. Preincisional ipsilateral stellate ganglion block for acute post operative pain control in unilateral mastectomy. PakJ Med Sci. 2011;27(4):879-83.

7. Hashemzadeh S, Hashemzadeh K, Hosseinzadeh H, Aligholipour Maleki R, Golzari SE. Comparison thoracic epidural and intercostal block to improve ventilation parameters and reduce pain in patients with multiple rib fractures. J Cardiovasc Thorac Res. 2011;3(3):87-91. doi:10.5681/jcvtr.2011.019. [PubMed: 24250961]

8. Soleimanpour H, Ghafouri RR, Taheraghdam A, Aghamohammadi D, Negargar S, Golzari SE, et al. Effectiveness of intravenous dexamethasone versus propofol for pain relief in the migraine headache: a prospective double blind randomized clinical trial. BMC Neurol. 2012;12:114. doi: 10.1186/1471-2377-12-114. [PubMed: 23020264]

9. Golzari SE, Soleimanpour H, Mahmoodpoor A, Safari S, Ala A. Lidocaine and pain management in the emergency department: a review article. Anesth Pain Med. 2014;4(1):e15444. doi: 10.5812 aapm.15444. [PubMed: 24660158]

10. Chung F, Ritchie E, Su J. Postoperative pain in ambulatory surgery. Anesth Analg. 1997;85(4):808-16. [PubMed: 9322460]

11. Brennan F, Carr DB, Cousins M. Pain management: a fundamental human right. Anesth Analg. 2007;105(1):205-21. doi: 10.1213/01. ane.0000268145.52345.55. [PubMed: 17578977]

12. White PF, Kehlet H, Neal JM, Schricker T, Carr DB, Carli F, et al. The role of the anesthesiologist in fast-track surgery: from multimodal analgesia to perioperative medical care. Anesth Analg. 2007:104(6):1380-96. doi: 10.1213/01.ane.0000263034.96885.e1. [PubMed: 17513630]

13. Beauregard L, Pomp A, Choiniere M. Severity and impact of pain after day-surgery. Can J Anaesth. 1998;45(4):304-11. doi: 10.1007| BF03012019. [PubMed: 9597202]

14. Rawal N. Analgesia for day-case surgery. BrJAnaesth.2001;87(1):7387. [PubMed: 11460815]

15. Rawal N, Hylander J, Nydahl PA, Olofsson I, Gupta A. Survey of postoperative analgesia following ambulatory surgery. Acta Anaesthesiol Scand.1997;41(8):1017-22. [PubMed: 9311400]

16. Roberts BL, Peterson GM, Friesen WT, Beckett WG. An investigation of pain experience and management following gynecologi- cal day surgery: differences between open and closed surgery. $J$ Pain Symptom Manage. 1995;10(5):370-7. [PubMed: 7673769]

17. Finley GA, McGrath PJ, Forward SP, McNeill G, Fitzgerald P. Parents' management of children's pain following 'minor' surgery. Pain. 1996;64(1):83-7. [PubMed: 8867249]

18. Zeidan A, Mazoit JX, Ali Abdullah M, Maaliki H, Ghattas T, Saifan A. Median effective dose $(\operatorname{ED}(5)(0))$ of paracetamol and morphine for postoperative pain: a study of interaction. Br J Anaesth. 2014;112(1):118-23. doi: 10.1093/bja/aet306. [PubMed:24157897]

19. Kolahdouzan K, Eydi M, Mohammadipour Anvari H, Golzari SE, Abri R, Ghojazadeh M, et al. Comparing the efficacy of intravenous acetaminophen and intravenous meperidine in pain relief after outpatient urological surgery. Anesth Pain Med. 2014;4(5):e20337. doi: 10.5812/aapm.20337. [PubMed: 25798377]

20. Schug SA, Sidebotham DA, McGuinnety M, Thomas J, Fox L. Acetaminophen as an adjunct to morphine by patient-controlled analgesia in the management of acute postoperative pain. Anesth Analg. 1998;87(2):368-72. [PubMed: 9706932]

21. Dahl JB, Kehlet $H$. The value of pre-emptive analgesia in the treatment of postoperative pain. Br J Anaesth. 1993;70(4):434-9. [PubMed: 8499204]

22. Breivik H. Postoperative pain management: why is it difficult to show that it improves outcome? EurJAnaesthesiol.1998;15(6):74851. [PubMed: 9884866]

23. Dahl V, Raeder JC. Non-opioid postoperative analgesia. Acta Anaesthesiol Scand. 2000;44(10):1191-203. [PubMed:11065198]

24. Hyllested M, Jones S, Pedersen JL, Kehlet H. Comparative effect of paracetamol, NSAIDs or their combination in postoperative pain management: a qualitative review. Br J Anaesth. 2002;88(2):199214. [PubMed: 11878654]

25. Farkas JC, Larrouturou P, Morin JP, Laurian C, Huchet J, Cormier $\mathrm{JM}$, et al. Analgesic efficacy of an injectable acetaminophen versus a dipyrone plus pitofenone plus fenpiverinium association after abdominal aortic repair. Curr Ther Res.1992;51(1):19-27.

26. Varrassi G, Marinangeli F, Agro F, Aloe L, De Cillis P, De Nicola A, et al. A Double-Blinded Evaluation of Propacetamol Versus Ketorolac in Combination with Patient-Controlled Analgesia Morphine. Anesth Analgesia.1999;88(3):611-6. doi:10.1097/00000539199903000-00028.

27. Farhat F, Savoyen MC, Jayr C. [Efficacy of propacetamol in postoperative pain based on two modes of intravenous administration]. Cah Anesthesiol. 1995;43(4):351-6. [PubMed: 8564652]

28. Zhou TJ, Tang J, White PF. Propacetamol versus ketorolac for treatment of acute postoperative pain after total hip or knee replacement. Anesth Analg. 2001;92(6):1569-75. [PubMed: 11375848]

29. Van Aken H, Thys L, Veekman L, Buerkle H. Assessing analgesia in single and repeated administrations of propacetamol for postoperative pain: comparison with morphine after dental surgery. Anesth Analg. 2004;98(1):159-65. [PubMed:14693612]

30. Sinatra RS, Jahr JS, Reynolds LW, Viscusi ER, Groudine SB, PayenChampenois C. Efficacy and safety of single and repeated administration of 1 gram intravenous acetaminophen injection (paracetamol) for pain management after major orthopedic surgery. Anesthesiology. 2005;102(4):822-31. [PubMed: 15791113]

31. Church CA, Stewart C, O. Lee T], Wallace D. Rofecoxib versus hydrocodone/acetaminophen for postoperative analgesia in functional endoscopic sinus surgery. Laryngoscope. 2006;116(4):6026. doi:10.1097/01.MLG.0000208341.30628.16. [PubMed:16585866]

32. Graham GG, Scott KF. Mechanism of action of paracetamol. Am J Ther. 2005;12(1):46-55. [PubMed: 15662292]

33. Pickering G, Loriot MA, Libert F, Eschalier A, Beaune P, Dubray C. Analgesic effect of acetaminophen in humans: first evidence of a central serotonergic mechanism. Clin Pharmacol Ther. 2006;79(4):371-8. doi: 10.1016/j.clpt.2005.12.307. [PubMed: 16580905]

34. Lucas R, Warner TD, Vojnovic I, Mitchell JA. Cellular mechanisms of acetaminophen: role of cyclo-oxygenase. FASEB J. 2005;19(6):635-7. doi:10.1096/fj.04-2437fje.[PubMed:15705740]

35. Ziemann-Gimmel P. Hypotension with acetaminophen-maybe there is a different mechanism. Aust Crit Care. 2011;24(3):150. doi: 10.1016/j.aucc.2011.04.003. [PubMed:21565519] 